hep-ph/9705411

DOE/ER/40561-325-INT97-00-171

UW/PT-97-12

BUHEP-97-15

\title{
Flavor from Strongly Coupled Supersymmetry
}

\author{
David B. Kaplan ${ }^{a}$, François Lepeintre ${ }^{a}$ and Martin Schmaltz ${ }^{b}$ \\ ${ }^{a}$ Institute for Nuclear Theory, Box 351550 \\ University of Washington, \\ Seattle, WA 98195-1550 \\ dbkaplan@phys.washington.edu, francois@u.washington.edu \\ ${ }^{b}$ Department of Physics \\ Boston University \\ Boston, MA 02215, USA \\ schmaltz@abel.bu.edu
}

\begin{abstract}
Strongly coupled supersymmetric theories can give rise to composite quarks and leptons at low energy. We show that the internal structure of these particles can explain the origin of three generations and provide a qualitative understanding of mass ratios and mixing angles between the different flavors of fermions, all within a renormalizable theory. The main point of the paper is to show how fermion masses and mixing angles can result from a "dual" Frogatt-Nielsen mechanism: fields neutral under $S U(3) \times S U(2) \times U(1)$ which carry flavor quantum numbers are confined within quarks and leptons, and from their perturbative interactions arises the observed flavor structure.
\end{abstract}




\section{Introduction}

The large hierarchy between the electroweak and Planck scales suggests that electroweak symmetry breaking is associated with nonperturbative physics in analogy with the hierarchy between $\Lambda_{Q C D} / M_{P}$. Various theoretical arguments also indicate that the world may be supersymmetric at short distances (see, for example, [1]). While supersymmetry (SUSY) protects the electroweak scale from radiative corrections and associates it with the SUSY breaking scale, it does not by itself explain the origin of the large hierarchy. Thus it is often suggested that a SUSY theory with nonperturbative dynamics lies behind the Standard Model (SM).

In much of the literature it is assumed that the nonperturbative SUSY physics lies in a hidden sector, decoupled from Standard Model particles [1]. It seems to us a rather strong assumption that nonperturbative physics exists at short distance solely to fix the electroweak scale (or the SUSY breaking scale), without affecting any other low energy observables. In this paper we speculate that strong interactions are not only responsible for the electroweak hierarchy but also for a substructure for quarks and leptons which could explain family replication, mass hierarchies and flavor mixing. Our motivation is partly opportunistic - there have been significant advances in the past several years in the understanding of strongly interacting SUSY theories [2, 3], and one can now construct models of composite quarks and leptons [4] with a degree of theoretical confidence impossible until recently. The development of new theoretical tools begs application to the old problem of flavor. As we discuss at length below, compositeness can provide a simple explanation for why families are exact replicas of each other, as far as $S U(3) \times S U(2) \times U(1)$ gauge charges are concerned, while being distinguished by their masses and mixing angles. An analogy can be made between the SM generations and nuclear isotopes. Consider, for example, the three isotopes of hydrogen: they each have the same chemistry yet have dramatically different masses - a fact simply understood once it is realized that the nucleus is composite, and that the three isotopes each contain a single proton but varying numbers of neutrons. Similarly, quarks and leptons could be bound states of both charged and neutral constituents, with different numbers or types of neutral constituents for the different generations. The nature of these composites will be determined by the underlying strong interactions, and the interactions of the "neutrons" will largely determine the flavor structure observed at low energies.

In this paper we show how a strongly coupled SUSY theory can realize this paradigm for the origin of three families, particle masses, and flavor mixing. We begin by discussing the replication of families. We then devise a new mechanism for flavor structure along the lines of the isotope analogy discussed above. We then describe several renormalizable models in which all quarks and leptons are composite, and which reproduce qualitatively the flavor structure we observe in the Standard Model. We conclude with speculations on future directions along the lines proposed here. 


\section{Family replication}

The first issue to be addressed is what sort of strongly coupled SUSY gauge theory to consider. A minimum requirement is that the theory must have composite particles in its spectrum which transform non-trivially under a sufficiently large symmetry group to contain $S U(3) \times S U(2) \times U(1)$. (In this work, we simplify our task by not trying to simultaneously explain flavor physics and dynamical SUSY breaking.) As discussed at length in [5], the properties of $N=1$ supersymmetric gauge theories without a tree level superpotential are largely determined by the number

$$
\chi \equiv \sum_{j} \mu_{j}-\mu(G)
$$

where $\mu_{j}$ and $\mu(G)$ are the Dynkin indices for the matter and adjoint representations respectively of the gauge group $G$. Normalizing $\mu=1$ for the fundamental representation, $\chi$ is even. Theories with $\mu(G)<\chi<0$ have runaway vacua and no groundstate or break supersymmetry. Theories with $\chi>2$ have moduli spaces of inequivalent vacua with massless gauge bosons at the origin; for many of these theories dual descriptions are known. Of the theories with $\chi=0$ or $\chi=2$ many are known to confine; in the case of $\chi=0$ confinement always occurs with a quantum deformed moduli space which breaks chiral symmetry, whereas confining $\chi=2$ theories have unbroken chiral symmetry at the origin of moduli space and dynamically generate a superpotential for the confined fields.

The $\chi<0$ theories do not appear promising to us; although the runaway vacua may be stabilized by superpotential terms, the minimum will typically be characterized by vacuum expectation values (VEVs) for fields that break the global symmetries, which we wish to preserve. The $\chi>2$ theories are interesting - one can imagine that the Standard Model is the dual of some strongly coupled theory, with the quarks and leptons being the magnetic degrees of freedom, and the SM gauge group being the dual gauge group after possible partial spontaneous symmetry breaking. However, working backward from the SM to find a dual typically leads to enormous gauge groups and we have yet to see or think of a clever approach of this type. The confining $\chi=2$ theories are particularly interesting for several reasons. First, they exhibit confinement and possess large unbroken global symmetries. Secondly, these "s-confining" theories, have been completely classified in [5], and their dynamically generated superpotentials can be constructed straightforwardly. We focus on these theories because they are the best understood and possess the properties we desire. (Confining $\chi=0$ theories might also be interesting even though — or because [6] the quantum deformed moduli space forces partial chiral symmetry breaking. Their properties can often be derived from the s-confining $\chi=2$ theories by giving a large mass to a flavor so that it decouples.)

A particularly intriguing example of an $s$-confining theory is an $S p(2 N)$ gauge theory with six fundamentals $Q$ and an antisymmetric tensor $A$ [7, B]. The theory 
has an $S U(6) \times U(1)$ global symmetry, as well as an $R$ symmetry. The confined description involves the $S p(2 N)$ neutral fields

$$
\begin{aligned}
T_{m} & =\operatorname{Tr} A^{m}, m=2,3, \ldots, N, \\
M_{n} & =Q A^{n} Q, n=0,1,2, \ldots, N-1,
\end{aligned}
$$

where $S p(2 N)$ indices are contracted with the appropriate metric, which can be taken to be $J=i \sigma_{2} \times 1_{N}$ 円. The quantum numbers of the fields are:

\begin{tabular}{c|c|ccc} 
& $S p(2 N)$ & $S U(6)$ & $U(1)$ & $U(1)_{R}$ \\
\hline$A$ & $\boxminus$ & 1 & -3 & 0 \\
$Q$ & $\square$ & $\square$ & $N-1$ & $\frac{1}{3}$ \\
\hline \hline$T_{m}$ & & 1 & $-3 m$ & 0 \\
$M_{n}$ & & $G$ & $2(N-1)-3 n$ & $\frac{2}{3}$
\end{tabular}

This model has a number of desirable features, and will be the "workhorse" of all the explicit models discussed below. If weak gauge interactions are embedded in the $S U(6)$ symmetry of this model, then there is a replication of "families" of $M$ fields [8]. Furthermore, in spite of having $N$ families, the family symmetry of the model is not $U(N)$, but only $U(1)$. Family replication arises because the $A$ field only carries this global $U(1)$ charge, and so the SM gauge charges of a composite particle are independent of the number of $A$ fields it contains. Breaking this $U(1)$ flavor symmetry will allow us to generate flavor in a manner analogous to the FroggattNielsen mechanism. The model realizes the isotope paradigm of the introduction, with the $(Q Q)$ and $A$ fields playing the roles of the proton and neutron respectively.

\section{A new mechanism for generating texture}

If all Yukawa interactions in the Standard Model were to vanish there would be a global $U(3)^{5}$ chiral flavor symmetry. The real Yukawa couplings explicitly break this chiral symmetry down to $U(1)_{B} \times U(1)_{L}$, but in a hierarchical manner, as apparent from the hierarchy of observed fermion masses and mixing angles. Effective low energy models of the symmetry breaking can be constructed: one makes assumptions about what subgroup $H \in U(3)^{5}$ is the approximate chiral symmetry at short distances, and then introduces spurions which break $H$ down to $U(1)_{B} \times U(1)_{L}$. The advantage of such models is that the large mass hierarchies we observe may be explained

\footnotetext{
${ }^{1}$ The number of families equals $N$ because the matrix $A J$ has eigenvalues which come in pairs; thus it satisfies the square root of its characteristic equation, implying that $A^{N+1}$ may be expressed in terms of lower powers of $A$.
} 
qualitatively in terms of several parameters of order $1 / 10$, however the analysis is never unique due to the paucity of information about the Yukawa matrices available to us experimentally. The analysis is further complicated in supersymmetric theories, where the physics of flavor and of SUSY breaking may be intertwined, as in general squark and slepton masses are sensitive to both.

To proceed, one must go beyond the effective description of flavor in terms of spurions, and construct models for the origin and communication of flavor symmetry breaking. It is possible that the origins of flavor lie above the Planck scale, or that the breaking of flavor symmetries is due to "Planck slop" — nonrenormalizable operators suppressed by powers of $M_{P}$. However, without a renormalizable theory of flavor, one gains little insight beyond that obtained from the spurion analysis.

A perturbative framework for generating flavor texture was proposed in [9] by Froggatt and Nielsen (FN). The proposal is that at short distance the only global flavor symmetry consists of $U(1)$ groups. Quarks and leptons are coupled to heavy, vector-like fields $V$, which in turn couple to a field $A$ with strength $g$, such that the product $g A$ can be consistently assigned $U(1)$ charges. (Either the $A$ is charged, or $g$ is a $U(1)$ spurion, or both.) When the heavy $V$ fields are integrated out of the theory, nonrenormalizable operators involving the quarks and leptons are generated, involving powers of $g A / M_{V}$. Below $M_{V}$, the $A$ field acquires a VEV, and the quantity $\epsilon \equiv g\langle A\rangle / M_{V}$ serves as the flavor spurion with which the Yukawa couplings are constructed. Various $\epsilon$ 's can be assigned charges under the $U(1)$ flavor symmetries, and the different elements of the Yukawa matrices are constrained by the quark and lepton charge assignments to be proportional to different powers of $\epsilon$ 10]. This framework can be generalized to incorporate nonabelian symmetries [11], both discrete and continuous.

The drawback of the FN approach is that whereas the 54 real parameters of the SM Yukawa matrices (in a particular basis) are traded for typically far fewer charges and VEVs, the charge assignments and symmetry breaking patterns tend to look quite ad hoc and little insight is gained into the origins of flavor.

The mechanism for generating flavor structure that we propose here is similar to the FN model, except that instead of having the $A$ field get an expectation value, we have it carry strong interactions that cause it to be confined within the quarks and leptons. Thus the spurion characterizing the flavor hierarchies is not $g\langle A\rangle / M_{V}$, but rather $\epsilon=g \Lambda / M_{V}$, where $\Lambda$ is the confinement scale of the quark and lepton constituents. Since the $A$ field is in a confined phase, instead of a Higgs phase as in the FN scenario, our mechanism is in some sense dual to the FN mechanism. The advantage of this approach is twofold: the FN charges of the quarks and leptons are now set by the number of $A$ constituents, determined by dynamics rather than fiat. Also, the two mass scales appearing in $\epsilon, M_{V}$ and $\Lambda$ are not entirely independent since typically the strong group will run much faster after the $V$ fields are integrated out, so that $\Lambda$ will not be far below $M_{V}$.

In the next section we give an example of a toy model which realizes the features 
we have been discussing - the low energy theory consists of composite fields for which both family replication and hierarchical Yukawa interactions arise as a consequence of the internal structure of the composites.

\section{$4 \quad$ A toy model with $S U(3)^{3}$ symmetry}

We now construct a model which illustrates the flavor mechanism discussed above. It is far from realistic - leptons end up being the heaviest particles, $u$ and $d$ quarks have proportional mass matrices, and the CKM matrix is trivial. Nevertheless, it nicely illustrates how nontrivial texture can arise dynamically in a renormalizable model where all SM particles are composite. In the subsequent section we will discuss more realistic models.

\subsection{Fundamental fields and interactions}

Yukawa interactions have the generic form $L H R$, where $L$ and $R$ correspond to the left- and right-handed fermions and $H$ is the Higgs. If all three fields are composite, such an interaction might be generated nonperturbatively due to instantons, in which case, following the power counting scheme of ref. [12], the Yukawa coupling would be $\sim 4 \pi$. Thus only the top Yukawa interaction can be due purely to strong dynamics, and we assume that the weaker Yukawa interactions arise from some interplay between strong and perturbative interactions. In the models we construct, $L$ and $R$ (with the exception of the top, in one example) are composed of constituents bound by different strong forces, and are able to interact only due to perturbative interactions. In the toy model we present here, we assume that the Higgs is composite as well, and thus that the strong interaction corresponds to a semisimple gauge group with three factors.

Since we wish to explain the existence of three families, we take the strong group to be $S p(6)^{3}=S p(6)_{L} \times S p(6)_{H} \times S p(6)_{R}$. To create composite SM fields we include a single antisymmetric tensor and six fundamentals for each $S p(6)$ factor, as in eq. (2.3). Without any perturbative superpotential added to the theory, the model possesses a global $S U(6)^{3}$ symmetry, in which we can embed "trinification", the $S U(3)^{3} \ltimes Z_{3}$ GUT introduced in [13] where the $Z_{3}$ symmetry cyclicly permutes the three $S U(3)$ group factors. A SM family is embedded in the "trinified" representation $(3, \overline{3}, 1) \oplus(1,3, \overline{3}) \oplus$ $(\overline{3}, 1,3)$. We take the fundamental "preons" to transform under $S p(6)^{3} \times S U(3)^{3}$ as ?:

\footnotetext{
${ }^{2}$ From here on we use the notation that strongly coupled fundamental fields are lowercase, while composite fields are in capitals.
} 


\begin{tabular}{c|ccc|ccc} 
preon & $S p(6)_{L}$ & $S p(6)_{H}$ & $S p(6)_{R}$ & $S U(3)_{1}$ & $S U(3)_{2}$ & $S U(3)_{3}$ \\
\hline$a_{1}$ & $\boxminus$ & 1 & 1 & 1 & 1 & 1 \\
$p_{1}$ & $\square$ & 1 & 1 & 3 & 1 & 1 \\
$q_{1}$ & $\square$ & 1 & 1 & 1 & $\overline{3}$ & 1 \\
\hline$a_{2}$ & 1 & $\boxminus$ & 1 & 1 & 1 & 1 \\
$p_{2}$ & 1 & $\square$ & 1 & 1 & 3 & 1 \\
$q_{2}$ & 1 & $\square$ & 1 & 1 & 1 & 3 \\
\hline$a_{3}$ & 1 & 1 & $\boxminus$ & 1 & 1 & 1 \\
$p_{3}$ & 1 & 1 & $\square$ & 1 & 1 & 3 \\
$q_{3}$ & 1 & 1 & $\square$ & $\overline{3}$ & 1 & 1
\end{tabular}

Each of the $S p(6)$ factors gives rise to composite fields as discussed in $\S 2$ : there are the $T_{2}=a^{2}$ and $T_{3}=a^{3}$ fields, which are neutral under $S U(3)^{3}$, as well as the composite fields shown below:

\begin{tabular}{c|ccc} 
composite & $S U(3)_{1}$ & $S U(3)_{2}$ & $S U(3)_{3}$ \\
\hline$\Phi^{(1)}=p_{1} q_{1}$ & 3 & $\overline{3}$ & 1 \\
$\Phi^{(2)}=p_{2} q_{2}$ & 1 & 3 & $\overline{3}$ \\
$\Phi^{(3)}=p_{3} q_{3}$ & $\overline{3}$ & 1 & 3 \\
\hline$X=q_{3} q_{3}$ & 3 & 1 & 1 \\
$\bar{X}=p_{1} p_{1}$ & $\overline{3}$ & 1 & 1 \\
$Y=q_{1} q_{1}$ & 1 & 3 & 1 \\
$\bar{Y}=p_{2} p_{2}$ & 1 & $\overline{3}$ & 1 \\
$Z=q_{2} q_{2}$ & 1 & 1 & 3 \\
$\bar{Z}=p_{3} p_{3}$ & 1 & 1 & $\overline{3}$
\end{tabular}

For simplicity we have only listed the family with no $a$ constituents; there are in fact three families. For example, the three families of $\Phi^{(1)}$ fields are $\left(p_{1} q_{1}\right),\left(p_{1} a_{1} q_{1}\right)$, and $\left(p_{1} a_{1}^{2} q_{1}\right)$. In this model, the fields with the most $a$ constituents will be the lightest, and so these composites correspond to the third, second, and first family respectively.

The SM gauge group is contained in $S U(3)^{3}$ by identifying $S U(3)_{c}=S U(3)_{1}$, while embedding $S U(2)_{w} \subset S U(3)_{2}$ and $U(1)_{Y} \subset S U(3)_{2} \times S U(3)_{3}$. With this embedding, the $\Phi$ fields decompose as

$$
\Phi^{(1)} \rightarrow Q \oplus G
$$




$$
\begin{aligned}
& \Phi^{(2)} \rightarrow L \oplus \bar{E} \oplus \bar{N} \oplus H_{u} \oplus H_{d} \oplus S \\
& \Phi^{(3)} \rightarrow \bar{U} \oplus \bar{D} \oplus \bar{G}
\end{aligned}
$$

where $Q, \bar{U}, \bar{D}, L, \bar{E}, H_{u, d}$ are fields with $\mathrm{SM}$ quantum numbers, $\bar{N}$ is a right-handed neutrino, $G$ is a $(3,1)_{-1 / 3}$ exotic diquark, and $S$ is a singlet. A virtue of this model is that all exotic fields are in real representations of the SM and can in principle acquire large masses.

We now add a perturbative superpotential so that the three sets of composite fields can interact with each other. The purpose of this exercise is to generate a superpotential in the low energy theory that includes a Yukawa interaction between composites of the three strong groups, which exhibits a hierarchical structure among the three families. To generate the desired interaction we introduce three heavy fields $v_{i}$, each of which transform as fundamentals under two of the strong $S p(6)$ groups:

\begin{tabular}{c|ccc|ccc} 
heavy field & $S p(6)_{L}$ & $S p(6)_{H}$ & $S p(6)_{R}$ & $S U(3)_{1}$ & $S U(3)_{2}$ & $S U(3)_{3}$ \\
\hline$v_{1}$ & 1 & $\square$ & $\square$ & 1 & 1 & 1 \\
$v_{2}$ & $\square$ & 1 & $\square$ & 1 & 1 & 1 \\
$v_{3}$ & $\square$ & $\square$ & 1 & 1 & 1 & 1
\end{tabular}

With these fields we write down a nongeneric, renormalizable superpotential consistent with an $S p(6)^{3} \times S U(3)^{3} \ltimes Z_{3}$ symmetry:

$$
\begin{aligned}
W & =\sum_{i=1,2,3} \frac{1}{2} \mathcal{M}_{i} v_{i}^{2}+\beta v_{1} v_{2} v_{3}+\gamma p_{i-1} v_{i} q_{i+1} \\
\mathcal{M}_{i} & \equiv\left(\mu-\alpha_{+} a_{i+1}-\alpha_{-} a_{i-1}\right) .
\end{aligned}
$$

where the subscripts are integers modulo $3, \mu$ is the common mass of the $v$ fields, and $\alpha_{ \pm}, \beta$ and $\gamma$ are coupling constants. This superpotential has the necessary couplings for the three sectors to communicate. Note that the $a v^{2}$ couplings break the $U(1)^{3}$ family symmetry that counts the $a$ fields, thus allowing mixing between families.

\subsection{The effective superpotential}

We integrate out the $v$ fields at their mass scale $\mu$, assumed to be above the confinement scale $\Lambda$, and expand the effective superpotential in powers of the coupling $\beta$. The result at lowest order arising from the contribution in fig. 1 is

$$
W_{0}=-\frac{\gamma^{2}}{2} \sum_{i}\left(p_{i+1} q_{i-1}\right)^{2} / \mathcal{M}_{i}
$$




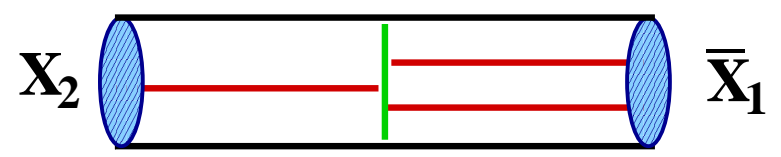

Fig 1. Contribution to the effective superpotential at order $\beta^{0}$ from integrating out the massive $\mathrm{v}$ fields. Exterior lines are the $\mathrm{p}$ and $\mathrm{q}$ preons; internal lines are $\mathrm{v}$ propagators, from which a preons are emitted. The $\mathrm{p}, \mathrm{q}$ and a preons later confine, and below the confinement scale this contribution to $W_{\text {eff }}$ is a mass term, where the number of a constituents determines the family numbers of the composites.

At the scale $\Lambda$, the theory confines and the operator (4.6) gets mapped onto a mass term for composite fields.

By expanding the propagators $1 / \mathcal{M}_{i}$ in eq. (4.6) in powers of each of the $a_{i}$ fields up to second order, we can compute the effective superpotential in terms of confined fields 5 . If we denote the families with subscripts $n=1,2,3$ such that $n=1$ corresponds to the lightest family (maximum number of $a$ constituents) while $n=3$ corresponds to the heaviest family (no $a$ constituents), then at order $\beta^{0}$ we find that the three families of real exotics $X, \bar{X}, Y, \bar{Y}, Z, \bar{Z}$ acquire hierarchical masses

$$
W_{0} \rightarrow\left(\frac{\Lambda^{2}}{\mu}\right) M_{m n}\left(\bar{X}_{m} X_{n}+\bar{Y}_{m} Y_{n}+\bar{Z}_{m} Z_{n}\right)
$$

where the mass matrix $M$ is given by $(r, s=0,1,2)$

$$
\begin{aligned}
M_{3-r, 3-s} & =\left.\left(\frac{\Lambda}{\mu}\right)^{r+s} \frac{1}{r ! s !} \partial_{x}^{r} \partial_{y}^{s}\left(1-\alpha_{+} x-\alpha_{-} y\right)^{-1}\right|_{x=y=0}=\frac{(r+s) !}{r ! s !} \epsilon_{+}^{r} \epsilon_{-}^{s} \\
& =\left(\begin{array}{ccc}
6 \epsilon_{-}^{2} \epsilon_{+}^{2} & 3 \epsilon_{-} \epsilon_{+}^{2} & \epsilon_{+}^{2} \\
3 \epsilon_{-}^{2} \epsilon_{+} & 2 \epsilon_{-} \epsilon_{+} & \epsilon_{+} \\
\epsilon_{-}^{2} & \epsilon_{-} & 1
\end{array}\right)
\end{aligned}
$$

with the definition

$$
\epsilon_{ \pm} \equiv\left(\frac{\Lambda}{\mu}\right) \alpha_{ \pm}
$$

While we are able to reliably calculate the effective superpotential we cannot determine the Kähler potential and with it the wave function renormalization for the composite fields. However, in the absence of small parameters in the strong dynamics we can assume that the relative wave function renormalization of the different composites differs at most by $\mathcal{O}(1)$ factors and does not wash out the predicted hierarchy.

At order $\beta$, the effective superpotential arises from the graph in fig. 2 , which yields

\footnotetext{
${ }^{3}$ In the following expressions we set to zero the $T$ moduli, the singlet fields composed entirely of $a$ constituents. It is not difficult to include them if one wishes.
} 


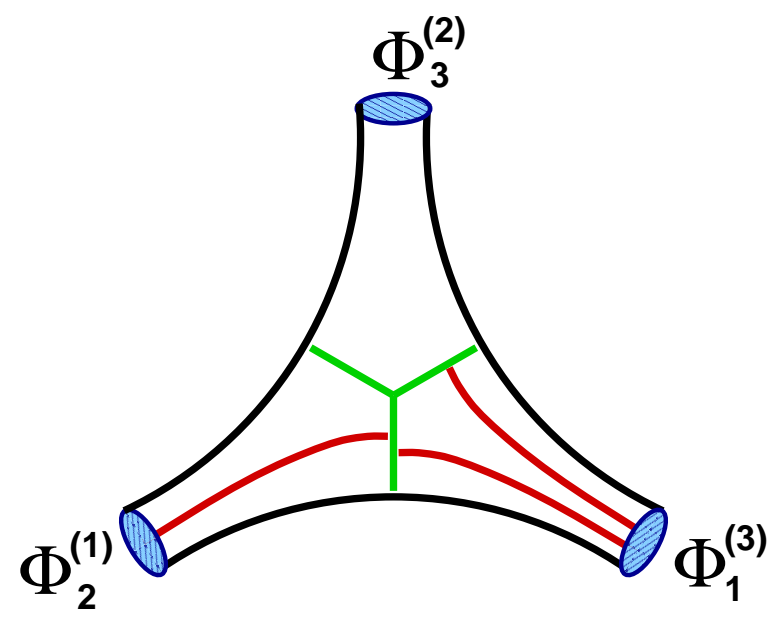

Fig 2. Contribution to the effective superpotential at order $\beta$ from integrating out the massive $\mathrm{v}$ fields. Below the confinement scale, these contributions become trilinear interactions between composites.

$$
\begin{aligned}
W_{1} & =-\beta \gamma^{3}\left(p_{1} q_{1}\right)\left(p_{2} q_{2}\right)\left(p_{3} q_{3}\right) /\left(\mathcal{M}_{1} \mathcal{M}_{2} \mathcal{M}_{3}\right) \\
& \rightarrow \beta\left(\frac{\Lambda}{\mu}\right)^{3} y_{r s t} \Phi_{r}^{(2)} \Phi_{s}^{(1)} \Phi_{t}^{(3)} .
\end{aligned}
$$

where the three families of chiral composite fields $\Phi^{(i)}$ interact via the Yukawa coupling

$$
\begin{aligned}
y_{3-r, 3-s, 3-t} & =\left(\frac{\Lambda}{\mu}\right)^{r+s+t} \frac{1}{r ! s ! t !} \times \\
\partial_{x}^{r} \partial_{y}^{s} \partial_{z}^{t}\left(\left(1-\alpha_{+} x-\alpha_{-} y\right)\right. & \left.\left.\times 1-\alpha_{+} y-\alpha_{-} z\right)\left(1-\alpha_{+} z-\alpha_{-} x\right)\right)\left.^{-1}\right|_{x=y=z=0} .
\end{aligned}
$$

For example, the Yukawa matrix $y_{3 m n}$ has entries

$$
y_{3 m n}=\left(\begin{array}{ccc}
\left(\epsilon_{+}+\epsilon_{-}\right)^{4}+3 \epsilon_{+}^{2} \epsilon_{-}^{2} & \left(\epsilon_{+}+\epsilon_{-}\right)^{3}+\epsilon_{+}^{2} \epsilon_{-} & \left(\epsilon_{+}+\epsilon_{-}\right)^{2}-\epsilon_{+} \epsilon_{-} \\
\left(\epsilon_{+}+\epsilon_{-}\right)^{3}+\epsilon_{+} \epsilon_{-}^{2} & \left(\epsilon_{+}+\epsilon_{-}\right)^{2}+\epsilon_{+} \epsilon_{-} & \left(\epsilon_{+}+\epsilon_{-}\right) \\
\left(\epsilon_{+}+\epsilon_{-}\right)^{2}-\epsilon_{+} \epsilon_{-} & \left(\epsilon_{+}+\epsilon_{-}\right) & 1
\end{array}\right)
$$

We need not continue the $\beta$ expansion any further, since at higher order in $\beta$ the effective superpotential contains at least four pairs of $p$ and $q$ preons, which translates into a nonrenormalizable interaction below the confinement scale.

These Yukawa interactions eq. (4.12) contribute to $u$ and $d$ quark masses, provided that a pair of the three families of $H_{u, d}$ can be identified with the MSSM Higgs fields. However, since the composite Higgs and lepton fields arise from the same strong group $S p(6)_{H}$, interactions among themselves arise purely from nonperturbative physics, and not from $v$ exchange. Thus, to address the question of Higgs and lepton masses, one must consider nonperturbative contributions to the superpotential. The nonperturbative superpotential has been worked out for $S p(6)$ in [7, 8]. In 
the notation of table (2.3), where subscripts denote the number of $a$ constituents, and the composites have not yet been rescaled to mass dimension 1 , it is given by

$$
W_{d y n}=\frac{1}{\Lambda^{7}}\left(\frac{1}{3} T_{2}^{2} M_{0}^{3}+\frac{1}{2} T_{3} M_{1} M_{0}^{2}-\frac{1}{2} T_{2} M_{0}^{2} M_{2}+\frac{1}{4} M_{0} M_{2}^{2}+\frac{1}{4} M_{1}^{2} M_{2}\right) .
$$

The $M$ fields are antisymmetric tensors of $S U(6)$, while $T$ fields are singlets; subscripts denote the number of $a$ constituents. Under the $S U(3)^{3}$ decomposition (4.2), $M^{3} \rightarrow$ $\left(\Phi^{(2)}\right)^{3}+\bar{Y} \Phi^{(2)} Y$ for the $S p(6)_{H}$ composites, etc. The SM content of the three $\Phi_{a}^{(2)}$ fields may be written as a $3 \times 3$ matrix

$$
\Phi_{a}^{(2)}=\left(\begin{array}{ccc}
L & H_{d} & H_{u} \\
S & \bar{N} & \bar{E}
\end{array}\right)_{a}
$$

where the dynamical interaction $\left[\Phi^{(2)}\right]^{3}$ has indices contracted with two $\epsilon$ tensors as in a determinant. Among the interactions one finds

$$
\Phi_{a}^{(2)} \Phi_{b}^{(2)} \Phi_{c}^{(2)}=S_{a} H_{u, b} H_{d, c}+H_{d, a} L_{b} \bar{E}_{c}+H_{u, a} L_{b} \bar{N}_{c}+\ldots
$$

Comparing the above expression with $W_{d y n}$ in eq. (4.13), it is interesting to note that in the vacuum where the modulus $S_{1}$ has a VEV while other moduli vanish, the three families of $H_{u, d}$ fields have a dynamically generated mass matrix which is rank 2:

$$
M_{H}^{2} \propto\left\langle S_{2}\right\rangle\left(\begin{array}{lll}
0 & 1 & 0 \\
1 & 0 & 0 \\
0 & 0 & 0
\end{array}\right) .
$$

Thus only the Higgs fields without an a constituent survive to the weak scale in this vacuum.

While the above mechanism is an interesting way to ensure that a single pair of Higgs fields survive to low energy, it has unwelcome phenomenological consequences. One is that the superpotential contains a global $S U(2)_{R}$ symmetry relating $\bar{U} \leftrightarrow \bar{D}$ and $\bar{N} \leftrightarrow \bar{E}$. Thus $u$ and $d$ quarks both have Yukawa interactions given by (4.12), their mass ratios are equal and mixing angles vanish. Furthermore, one sees that the dynamically generated lepton mass matrices are rank 1 with one family being very massive (heavier than the top quark, as the $\beta(\Lambda / \mu)^{3}$ suppression appearing in eq. (4.10) is absent) while the other two lepton families are massless.

\subsection{Comments}

The model we have presented here is certainly no candidate for beyond the SM physics; however, it does provide an example of how a renormalizable field theory

\footnotetext{
${ }^{4}$ Above in eq. $((4.13))$ the subscripts denote the number of $a$ constituents; for the remainder of the paper subscripts will denote family number, defined as (3-number of $a$ constituents).
} 
can give rise to three composite families of SM fields with nontrivial flavor texture. The structure of the mass and Yukawa matrices of eqs. (4.8), (4.12) is sufficiently complex that one can imagine more sophisticated models based on our mechanism being at least in qualitative agreement with the flavor structure of the SM. This is the subject of the next section.

We conclude this section with a comment on the factor $\Lambda / \mu$ that appears in eq. (4.10), and which phenomenologically cannot be be allowed to be very small. Recall that $\Lambda$ characterizes the confinement scale, while $\mu$ is the mass of the $v$ fields that communicate between the Higgs and the left- and right-handed fields. One might worry about tuning a perturbative coupling to be close to a nonperturbative scale. However, note that the fundamental parameters of the theory in the ultraviolet are not $\mu$ and $\Lambda$, but rather $\mu$ and $\Lambda_{U V}$, where the latter is the scale that determines the running of the $S p(6)$ interactions above the scale $\mu$. The relation between $\Lambda_{U V}$ and the confining scale $\Lambda$ is easily estimated from the one-loop beta functions, and one finds in the present theory that

$$
\left(\frac{\Lambda}{\mu}\right)=\left(\frac{\Lambda_{U V}}{\mu}\right)^{1 / 7} .
$$

Thus, for example, on could have $\Lambda_{U V} / \mu$ vary from $10^{-3}$ to $10^{-2}$, while $\Lambda / \mu$ only varies between 0.4 and 0.5 . The lack of fine tuning reflects the fact that the $S p(6)$ groups are nearly asymptotically flat with the $v$ fields included, but confine quickly once the $v$ 's are integrated out.

\section{More realistic models}

In this section we construct two models based on the $S p(6)$ confining theory, which succeed in reproducing qualitatively much of the flavor structure seen in the SM. The purpose of these models is to show that a renormalizable, strongly coupled theory can give rise to nontrivial CKM angles and fermion mass ratios. The structure of model 1 is similar to the toy model of the previous section but with a cure for most of the shortcomings of the simpler toy. It possesses the interesting feature that the top Yukawa coupling is generated nonperturbatively from $S p(6)$ instanton interactions. However, the model cannot reproduce realistic masses and mixing angles. Model 2 is of slightly different structure and succeeds in fitting all the masses and mixing angles of the Standard Model. Both models are rather complicated, and are intended to serve as existence proofs rather than as paragons of beauty.

\section{1 $S p(6)^{3}$ with composite Higgs fields}

The main drawbacks of the toy model of the previous section were that (i) there were no CKM angles; (ii) the top quark Yukawa coupling was of order $\beta(\Lambda / \mu)^{3}$, which is 
likely to be too small; and (iii) the lepton masses were generated dynamically, and were therefore either too large or zero. The first model we examine is similar to the example of the previous section, but is designed to correct the three major deficiencies. In particular, the up Yukawa matrix receives both dynamical and perturbative contributions, allowing the top to be very heavy. The down type couplings are only generated perturbatively, and so they are naturally light. As up and down sectors are now treated differently, nontrivial mixing angles and dissimilar mass ratios are possible. Finally, lepton masses are also generated perturbatively, but at higher order than the down quarks, so that they are naturally lighter. The price for these successes is that the model has less symmetry and is more complicated. It makes gauge coupling unification look mysterious, and it predicts incorrect relations, such as $m_{\mu} / m_{e} \sim 1700$, and $V_{u s} \sim V_{c b}$.

The strong group is taken to be $S p(6)^{3}$, and we take the same preons as in the previous example, with the exception that there is no $S U(3)^{3}$ symmetry, and the preons have different $U(1)$ assignments. These $U(1)$ assignments are unique under the requirement that (i) it is possible for the top quark Yukawa coupling to arise nonperturbatively, and (ii) that all charged exotics are real under $S U(3) \times S U(2) \times$ $U(1)$ so that they can in principle be heavy.

\begin{tabular}{c|ccc|rrrr} 
preon & $S p(6)_{L}$ & $S p(6)_{H}$ & $S p(6)_{R}$ & $S U(3)$ & $S U(2)$ & $U(1)_{Y}$ & $U(1)_{B-L}$ \\
\hline$a_{1}$ & $\boxminus$ & 1 & 1 & 1 & 1 & 0 & 0 \\
$t_{1}$ & $\square$ & 1 & 1 & 3 & 1 & $-1 / 3$ & $-1 / 6$ \\
$d_{1}$ & $\square$ & 1 & 1 & 1 & 2 & $1 / 2$ & $1 / 2$ \\
$s_{1}$ & $\square$ & 1 & 1 & 1 & 1 & 0 & $-1 / 2$ \\
\hline$a_{2}$ & 1 & $\boxminus$ & 1 & 1 & 1 & 0 & 0 \\
$d_{2}$ & 1 & $\square$ & 1 & 1 & 2 & $-1 / 2$ & $-1 / 2$ \\
$s_{21}$ & 1 & $\square$ & 1 & 1 & 1 & 0 & $-1 / 2$ \\
$s_{22}$ & 1 & $\square$ & 1 & 1 & 1 & 0 & $1 / 2$ \\
$s_{22}^{\prime}$ & 1 & $\square$ & 1 & 1 & 1 & 0 & $1 / 2$ \\
$s_{23}$ & 1 & $\square$ & 1 & 1 & 1 & 1 & $1 / 2$ \\
\hline$a_{3}$ & 1 & 1 & $\square$ & 1 & 1 & 0 & 0 \\
$t_{3}$ & 1 & 1 & $\square$ & $\overline{3}$ & 1 & $1 / 3$ & $1 / 6$ \\
$s_{31}$ & 1 & 1 & $\square$ & 1 & 1 & 0 & $-1 / 2$ \\
$s_{32}$ & 1 & 1 & $\square$ & 1 & 1 & 0 & $1 / 2$ \\
$s_{33}$ & 1 & 1 & $\square$ & 1 & 1 & -1 & $-1 / 2$
\end{tabular}

The first subscript on the preon fields designates under which $S p(6)$ group they transform. As before, "a" designates an $S p(6)$ antisymmetric tensor, while "s", "d", and "t" label $S p(6)$ fundamentals which transform as singlets, doublets, and triplets re- 
spectively under $S U(3) \times S U(2)$.

As before, the $S p(6)^{3}$ groups confine (assumed for simplicity to occur at the same scale) and the composite fields transform as the three families of the SM (with righthanded neutrinos), plus exotic states which are all real under $S U(3) \times S U(2) \times U(1)$. Their quantum numbers are

\begin{tabular}{|c|c|c|c|c|c|}
\hline con & nposite & $S U(3)$ & $S U(2)$ & $U(1)_{Y}$ & $U(1)_{B-L}$ \\
\hline $\bar{U}^{(1)}$ & $=t_{1} t_{1}$ & $\overline{3}$ & 1 & $-2 / 3$ & $-1 / 3$ \\
\hline $\bar{E}^{(1)}$ & $=d_{1} d_{1}$ & 1 & 1 & 1 & 1 \\
\hline & $=t_{1} d_{1}$ & 3 & 2 & $1 / 6$ & $1 / 3$ \\
\hline & $=t_{1} s_{1}$ & 3 & 1 & $-1 / 3$ & $-2 / 3$ \\
\hline$H_{u}^{(1)}$ & $=d_{1} s_{1}$ & 1 & 2 & $1 / 2$ & 0 \\
\hline$E^{(2)}$ & $=d_{2} d_{2}$ & 1 & 1 & -1 & -1 \\
\hline & $=d_{2} s_{21}$ & 1 & 2 & $-1 / 2$ & -1 \\
\hline$H_{d}^{(2)}$ & $=d_{2} s_{22}$ & 1 & 2 & $-1 / 2$ & 0 \\
\hline$H_{d}^{\prime(2)}$ & $=d_{2} s_{22}^{\prime}$ & 1 & 2 & $-1 / 2$ & 0 \\
\hline$H_{u}^{(2)}$ & $=d_{2} s_{23}$ & 1 & 2 & $1 / 2$ & 0 \\
\hline$S^{(2)}$ & $=s_{21} s_{22}$ & 1 & 1 & 0 & 0 \\
\hline$S^{\prime(2)}$ & $=s_{21} s_{22}^{\prime}$ & 1 & 1 & 0 & 0 \\
\hline$\phi_{+}$ & $=s_{21} s_{23}$ & 1 & 1 & 1 & 0 \\
\hline $\bar{N}$ & $=s_{22} s_{22}^{\prime}$ & 1 & 1 & 0 & 1 \\
\hline $\bar{E}^{(2)}$ & $=s_{22} s_{23}$ & 1 & 1 & 1 & 1 \\
\hline $\bar{E}^{\prime(2)}$ & $=s_{22}^{\prime} s_{23}$ & 1 & 1 & 1 & 1 \\
\hline$U$ & $=t_{3} t_{3}$ & 3 & 1 & $2 / 3$ & $1 / 3$ \\
\hline $\bar{D}$ & $=t_{3} s_{31}$ & $\overline{3}$ & 1 & $1 / 3$ & $-1 / 3$ \\
\hline $\bar{G}$ & $=t_{3} s_{32}$ & $\overline{3}$ & 1 & $1 / 3$ & $2 / 3$ \\
\hline $\bar{U}^{(3)}$ & $=t_{3} s_{33}$ & $\overline{3}$ & 1 & $-2 / 3$ & $-1 / 3$ \\
\hline$S^{(3)}$ & $=s_{31} s_{32}$ & 1 & 1 & 0 & 0 \\
\hline$E^{(3)}$ & $=s_{31} s_{33}$ & 1 & 1 & -1 & -1 \\
\hline$\phi_{-}$ & $=s_{32} s_{33}$ & 1 & 1 & -1 & 0 \\
\hline
\end{tabular}

The charge assignments have been made so that the first set of preons yields the composites $Q, \bar{U}^{(1)}$ and $H_{u}^{(1)}$, which have the right quantum numbers to generate a large dynamical top quark Yukawa coupling.

As before, we will add massive $v$ fields to the theory, which will generate perturbative preon interactions which will in turn become SM Yukawa interactions below the confinement scale $\Lambda$. Before specifying exactly what $v$ fields are needed, we sketch out how the various composites get their masses. 


\subsubsection{The $d$ quarks}

Since $Q, H_{d} / H_{d}^{\prime}$, and $\bar{D}$ fields arise from the three different $S p(6)$ groups, Yukawa interactions for the $d$ quarks can be generated via the interaction in fig. 2 , as in the model of the previous section. This will require three $v$ fields, which we denote $v_{1,2,3}$. The $d$ Yukawa coupling will then arise from expanding $1 /\left(\mathcal{M}_{1} \mathcal{M}_{2} \mathcal{M}_{3}\right)$, where $\mathcal{M}_{i}$ is the $a$ dependent mass of $v_{i}$, and thus is of the form (4.11), (4.12).

\subsubsection{The $u$ quarks}

There are six $\bar{U}$ fields in the theory (the three families of $\bar{U}^{(1)}$ and $\bar{U}^{(3)}$ ); three can get large masses by pairing up with the three $U$ fields. The mass matrix takes the form

$$
\begin{gathered}
\bar{U}^{(1)} \\
M_{U} \sim U\left(1 / \bar{U}_{2}(3)\right. \\
\left.4 \pi\left\langle S^{(3)}\right\rangle\right) .
\end{gathered}
$$

The factor of $4 \pi$ follows from the power counting arguments in ref. [12]. The coupling between $U$ and $\bar{U}^{(1)}$ arises through the exchange of the $v_{2}$ field proportional to $1 / \mathcal{M}_{2}$ and resembles the mass matrix $M_{m n}$ in eq. (4.8). The $S^{(3)}$ dependent coupling between $U$ and $\bar{U}^{(3)}$ arises from the nonperturbative potential (4.13) as discussed in $\S 4.2$, and its family structure is

$$
\left\langle S^{(3)}\right\rangle \propto\left(\begin{array}{ccc}
S_{3}^{(3)} & S_{2}^{(3)} & S_{1}^{(3)} \\
S_{2}^{(3)} & S_{1}^{(3)} & 0 \\
S_{1}^{(3)} & 0 & 0
\end{array}\right)
$$

Since $1 / \mathcal{M}_{2}$ is rank 3 , all three families of $U$ aquire masses. As it has the hierarchical structure seen in eq. (4.8), the $\bar{U}^{(1)}$ field that couples most strongly to $U$ is the one with no $a_{1}$ constituents. By choosing only $S_{1}^{(3)}$ to get a $\mathrm{VEV},\left\langle S^{(3)}\right\rangle$ is rank 2 , and by adjusting its size relative to $1 / \mathcal{M}_{2}$, one can arrange to have the $U$ quarks pair in such a way that the massless $\bar{U}$ quarks include one which is primarily $\bar{U}^{(1)}$ (the top), one which is entirely $\bar{U}^{(3)}$ (the up), and one which is mostly $\bar{U}^{(3)}$ (the charm). The $\bar{U}^{(3)}$ components can couple to an $H_{u}^{(2)}$ field through a perturbative diagram as in fig. 2, while the $\bar{U}^{(1)}$ components can couple nonperturbatively to $H_{u}^{(1)}$. Therefore we must have some linear combination of $H_{u}^{(1)}$ and $H_{u}^{(2)}$ develop a VEV at the electroweak scale.

\subsubsection{The $H_{u, d}$ doublets}

There are a total of six $H_{u, d}$ pairs in this theory, and we will assume that only one pair remains light down to the weak scale. Without loss of generality, we can take the light down Higgs to be in one of the three families of $H_{d}^{(2)}$ (as opposed to ${H^{\prime}}_{d}^{(2)}$ ). 


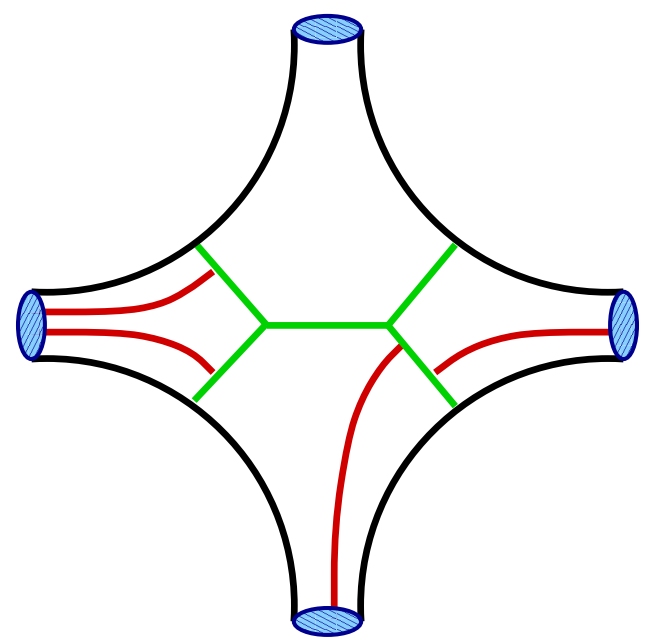

Fig 3. Contribution to the effective superpotential at order $\beta^{2}$ from integrating out the massive $v$ fields. Below the confinement scale, these contributions become quadrilinear interactions between composites.

From the previous discussion, we see that the light $H_{u}$ must have components in both $H_{u}^{(1)}$ (to give mass to the top) and in $H_{u}^{(2)}$ (to give mass to the up and charm). The Higgs mass matrix allows both $H_{d}^{(2)}$ and ${H^{\prime}}_{d}^{(2)}$ to couple perturbatively to $H_{u}^{(1)}$, and nonperturbatively to $H_{u}^{(2)}$. We can get the required mass pattern if we take the Higgs mass matrix to look like

$$
M_{H} \sim H_{u}^{(1)} H_{u}^{(2)}\left(\begin{array}{cc}
H_{d}^{(2)} & H_{d}^{\prime(2)} \\
0 & 1 / \mathcal{M}_{h} \\
4 \pi\left\langle{S^{\prime}}^{(2)}\right\rangle_{2} & 4 \pi\left\langle S^{(2)}\right\rangle_{3}
\end{array}\right)
$$

where $1 / \mathcal{M}_{h}$ is once again the sort of matrix in eq. (4.8) due to the exchange of a new $v_{h}$ field, and the subscripts on the $S$ VEVs signify the rank. Evidently, there is a single massless down-type Higgs which is $H_{d}^{(2)}$ (third family), and therefore there is a single massless up-type Higgs field. The latter contains components both in $H_{u}^{(1)}$ (primarily first family) and in $H_{u}^{(2)}$, as desired.

The fact that a pair of Higgs fields remains massless relies on the $H_{u}^{(1)}-H_{d}^{(2)}$ element in the matrix (5.5) remaining zero to all orders in perturbation theory. A potential problem might arise from $\mathcal{O}\left(\beta^{2}\right)$ contributions to $W_{\text {eff }}$ shown in fig. 3 , which could yield the effective operator $H_{u}^{(1)} H_{d}^{(2)} S^{(3)} S^{\prime(2)}$, for example, where the two singlets $S^{(3)}$ and $S^{\prime(2)}$ get VEVs. However, it is possible to avoid these contributions by choosing the couplings of the heavy $v$ fields appropriately, so that the zero in (5.5) is preserved at all orders in $W_{\text {eff }}$. 


\subsubsection{Charged leptons}

The charged lepton mass matrix takes the form

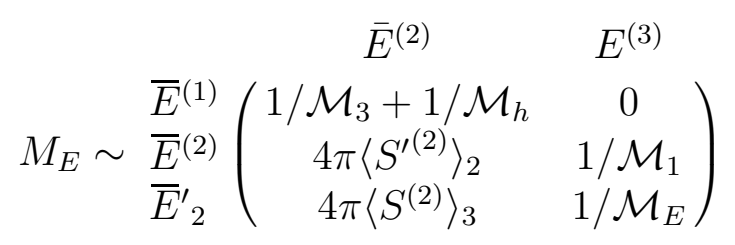

where $1 / \mathcal{M}_{1}, 1 / \mathcal{M}_{3}, 1 / \mathcal{M}_{h}$, and $1 / \mathcal{M}_{E}$ arise from exchange of $v_{1}, v_{3}, v_{h}$, and a new field $v_{E}$ respectively. The ranks of the nonperturbative contributions $\left\langle S^{(2)}\right\rangle,\left\langle{S^{\prime}}^{(2)}\right\rangle$ were fixed when considering the Higgs matrix above.

The massless $\bar{E}$ fields will be family dependent combinations of $\bar{E}^{(1)}, \bar{E}^{(2)}$, and $\bar{E}^{\prime(2)}$. Computing the Yukawa couplings to the light Higgs $H_{d, 0}^{(2)}$ of these fields is complicated, as the coupling to $L H_{d}^{(2)}$ is nonperturbative $(\sim 4 \pi)$ for $\bar{E}^{\prime(2)}$, zero for $\bar{E}^{(2)}$, and perturbative at order $\beta^{2}$ for $\bar{E}^{(1)}$ via the graph pictured in fig. 3. The $1 / \mathcal{M}_{E}$ term was introduced in eq. (5.6) so that the leptons would not contain much $\bar{E}^{\prime(2)}$, which would lead to excessively large lepton masses.

\subsubsection{Neutrinos}

As it stands, the model predicts that neutrino masses are similar to quark masses. The situation can be remedied by giving the right handed neutrinos a large mass so that the light neutrinos are predominantly left-handed with small Majorana masses given by the see-saw formula

$$
M_{\nu}=-\left[Y^{l}\right]^{T} M_{N}^{-1} Y^{l}\left\langle H_{u}\right\rangle^{2} .
$$

where $M_{N}$ is the Majorana mass matrix of the right handed neutrinos. Since the right handed neutrinos are composite as well their masses must arise from preon dynamics. A suitable mechanism involves adding an extra massive field $w_{N}$ with lepton number 1 which transforms as an antisymmetric tensor of the $S p(6)_{H}$ group. We include the following superpotential couplings

$$
W_{N}=\frac{1}{2}\left(\mu-\alpha_{N} a_{2}\right) w_{N}^{2}+w_{N} s_{22} s_{22}^{\prime} .
$$

Note that the mass term for $w_{N}$ breaks lepton number by two units as required for generating a Majorana mass for neutrinos. Integrating out $w_{N}$ yields a graph as in fig. 1. Expanding in $\alpha_{N}$, and matching onto confined fields gives the Majorana mass matrix for right-handed neutrinos

$$
M_{N} \sim \frac{\Lambda^{2}}{\mu}\left(\begin{array}{ccc}
6 \epsilon_{N}^{4} & 3 \epsilon_{N}^{3} & \epsilon_{N}^{2} \\
3 \epsilon_{N}^{3} & 2 \epsilon_{N}^{2} & \epsilon_{N} \\
\epsilon_{N}^{2} & \epsilon_{N} & 1
\end{array}\right), \quad \epsilon_{N}=\alpha_{N} \frac{\Lambda}{\mu} .
$$


The resulting masses for the left-handed neutrinos are easily determined because the dynamically generated Dirac mass matrix for the neutrinos has only one non-zero entry in the $(1,1)$ component. Thus the electron neutrino is massive with a mass that is inversely proportional to the compositeness scale $m_{\nu_{e}} \sim\left\langle H_{u}\right\rangle^{2} / \Lambda$, and the other two neutrino species are massless.

\subsubsection{Numerics}

A summary of the massive fields needed to generate the Yukawa interactions in this model are as follows:

\begin{tabular}{c|ccc} 
field & $S p(6)_{L}$ & $S p(6)_{H}$ & $S p(6)_{R}$ \\
\hline$v_{1}$ & 1 & $\square$ & $\square$ \\
$v_{2}$ & $\square$ & 1 & $\square$ \\
$v_{3}$ & $\square$ & $\square$ & 1 \\
$v_{E}$ & 1 & $\square$ & $\square$ \\
$v_{G}$ & $\square$ & 1 & $\square$ \\
$v_{H}$ & $\square$ & $\square$ & 1 \\
$w_{N}$ & 1 & $\boxminus$ & 1
\end{tabular}

The $v_{1,2,3}$ fields generate the $d$ quark Yukawa interactions as in the toy model of the previous section; they also play a role in the $u$ quark Yukawa interactions, along with the $S p(6)_{L}$ dynamical superpotential. The fields $v_{E, G, H}$ differ from $v_{1,2,3}$ in that they do not participate in $v^{3}$ interactions, and so can only generate masses for composites in real representations, as in fig. 1 ; they also couple to different combinations of the $s, d, t$ preons. For example, forbidding a coupling of either $v_{2}$ and $v_{3}$ to the preon $s_{1}$ (a constituent of $H_{u}^{(1)}$ ) protects the zero in the Higgs matrix (5.5) from contributions pictured in fig. 3 ; however, $s_{1}$ is also a constituent of $G$, and so $v_{G}$ must couple to it or else the $G$ and $\bar{G}$ composities will remain massless.

We give here a crude numerical fit to data, purposefully not fine tuned. We take the six $v$ fields to be degenerate, with the following couplings:

$$
\begin{array}{ll} 
& v_{1}\left[s_{33} s_{23}+s_{31} s_{22}+s_{21} s_{32}\right]-0.4\left(v_{1}^{2} / 2\right)\left[a_{2}+a_{3}\right] \\
+ & v_{2} t_{1} t_{3}-0.4\left(v_{2}^{2} / 2\right)\left[a_{1}+a_{3}\right] \\
+ & v_{3} d_{1} d_{2}+\left(v_{3}^{2} / 2\right)\left[0.3 a_{1}+0.7 a_{2}\right] \\
+ & 12 v_{E}\left[s_{31} s_{22}^{\prime}+s_{33} s_{23}\right]-0.9\left(v_{E}^{2} / 2\right)\left[a_{2}+a_{3}\right] \\
+ & v_{G}\left[0.1 t_{1} t_{3}+s_{1} s_{32}\right]-0.4\left(v_{G}^{2} / 2\right)\left[a_{1}+a_{3}\right] \\
+ & v_{H}\left[d_{1} d_{2}+s_{1} s_{22}^{\prime}\right]-0.3\left(v_{H}^{2} / 2\right)\left[a_{1}+a_{2}\right]
\end{array}
$$

The scalar VEVs are

$$
\frac{\left\langle S_{1}^{(2)}\right\rangle}{\Lambda}=-0.05 \frac{\Lambda}{4 \pi \mu}, \quad \frac{\left\langle\left(S_{2}^{\prime(2)}\right)\right\rangle}{\Lambda}=-0.03 \frac{\Lambda}{4 \pi \mu}
$$




$$
\frac{\left\langle S_{2}^{(3)}\right\rangle}{\Lambda}=0.03 \frac{\Lambda}{4 \pi \mu}, \quad \frac{\left\langle S_{3}^{(3)}\right\rangle}{\Lambda}=10 \frac{\Lambda}{4 \pi \mu}
$$

with

$$
\frac{\Lambda}{\mu}=\frac{1}{2}
$$

With these parameters one finds the quark and lepton mass ratios (at the scale $\Lambda$, which will be high),

$$
\begin{aligned}
m_{t} / m_{c}=410, & m_{c} / m_{u}=300, \\
m_{b} / m_{s}=24, & m_{s} / m_{d}=34, \\
m_{\tau} / m_{\mu}=15, & m_{\mu} / m_{e}=1700 \\
m_{b} / m_{\tau}=1 . &
\end{aligned}
$$

The CKM matrix (again computed at the scale $\Lambda$ ) is

$$
V_{C K M}=\left(\begin{array}{ccc}
0.98 & 0.16 & 0.07 \\
-0.17 & 0.97 & 0.17 \\
-0.05 & -0.18 & 0.98
\end{array}\right)
$$

Aside from displaying sophisticated flavor structure, we consider this model's interesting features to include a dynamically generated top mass, as well as the complex mixing between SM particles and vectorlike fields. The model also displays the pitfalls generic to theories of composite quarks and leptons that do not possess a baryon number symmetry, even if they are invariant under $B-L$ parity: in this model, dimension five proton decay operators are generated upon integrating out the massive $G$ field. We have not examined these operators in detail, but the proton lifetime is expected to be shorter than observed for any value of the compositeness scale below the Planck scale.

\subsection{A realistic model with fundamental Higgs fields}

By taking the Higgs fields to be fundamental instead of composite, we can simultaneously simplify the model and make it agree quantitatively with the observed world. In this section, we present an explicit example of such a model which successfully fits all masses and mixing angles of the SM. In this model the three generations of matter fields are composites of two strong $S p(6)$ groups which confine at a scale $\Lambda$ as described in $\S 2$. An interesting feature of this model is that baryon number is preserved exactly, thus preventing dangerous proton decay and allowing the compositeness scale to be low enough that there are experimentally testable consequences. 


\subsubsection{Field content}

We take the preons of the ultraviolet to transform under the Standard Model gauge group with well-defined baryon and lepton number.

\begin{tabular}{c|cc|rrrrr} 
preon & $S p(6)_{1}$ & $S p(6)_{2}$ & $S U(3)$ & $S U(2)$ & $U(1)_{Y}$ & $U(1)_{B}$ & $U(1)_{L}$ \\
\hline$a_{1}$ & $\boxminus$ & 1 & 1 & 1 & 0 & 0 & 0 \\
$p_{1}$ & $\square$ & 1 & 3 & 1 & $1 / 6$ & $-1 / 6$ & $-1 / 2$ \\
$q_{1}$ & $\square$ & 1 & 1 & 2 & 0 & $1 / 2$ & $1 / 2$ \\
$r_{1}$ & $\square$ & 1 & 1 & 1 & $-1 / 2$ & $-1 / 2$ & $1 / 2$ \\
\hline$a_{2}$ & 1 & $\square$ & 1 & 1 & 0 & 0 & 0 \\
$p_{2}$ & 1 & $\square$ & $\overline{3}$ & 1 & $-1 / 6$ & $1 / 6$ & $1 / 2$ \\
$q_{2}$ & 1 & $\square$ & 1 & 1 & $1 / 2$ & $-1 / 2$ & $-1 / 2$ \\
$r_{2}$ & 1 & $\square$ & 1 & 1 & $-1 / 2$ & $-1 / 2$ & $-1 / 2$ \\
$s_{2}$ & 1 & $\square$ & 1 & 1 & $1 / 2$ & $1 / 2$ & $-1 / 2$
\end{tabular}

After confinement of the two $S p(6)$ groups we obtain three generations of composite quarks and leptons, distinguished by the number of $a$ constituents. The Standard Model quantum numbers of these composites are

\begin{tabular}{c|crrrr} 
composite & $S U(3)$ & $S U(2)$ & $U(1)_{Y}$ & $U(1)_{B}$ & $U(1)_{L}$ \\
\hline$Q=p_{1} q_{1}$ & 3 & 2 & $1 / 6$ & $1 / 3$ & 0 \\
$\bar{U}=p_{2} r_{2}$ & $\overline{3}$ & 1 & $-2 / 3$ & $-1 / 3$ & 0 \\
$\bar{D}=p_{2} q_{2}$ & $\overline{3}$ & 1 & $1 / 3$ & $-1 / 3$ & 0 \\
$L=q_{1} r_{1}$ & 1 & 2 & $-1 / 2$ & 0 & 1 \\
$\bar{E}=q_{2} s_{2}$ & 1 & 1 & 1 & 0 & -1 \\
$\bar{N}=r_{2} s_{2}$ & 1 & 1 & 0 & 0 & -1 \\
$G=p_{2} p_{2}$ & 3 & 1 & $-1 / 3$ & $1 / 3$ & 1 \\
$\bar{G}=p_{1} p_{1}$ & $\overline{3}$ & 1 & $1 / 3$ & $-1 / 3$ & -1 \\
$R=p_{2} s_{2}$ & $\overline{3}$ & 1 & $1 / 3$ & $2 / 3$ & 0 \\
$\bar{R}=p_{1} r_{1}$ & 3 & 1 & $-1 / 3$ & $-2 / 3$ & 0 \\
$S=q_{1} q_{1}$ & 1 & 1 & 0 & 1 & 1 \\
$\bar{S}=q_{2} r_{2}$ & 1 & 1 & 0 & -1 & -1
\end{tabular}

where again we have only shown only one of three composite families; the remaining families contain one or two a preons.

In addition to the three SM families, there are three generations of right handed neutrinos, as well as exotics in real representations of both the Standard Model gauge 
group and baryon and lepton number. As advertised, the SM Higgs fields don't appear in the composite spectrum, they are added as fundamental fields.

At this stage there are no couplings of the composites to the Higgs fields, thus there are no SM Yukawa couplings. The dynamically generated superpotential (4.13) of the confining $S p(6)$ groups only couples composites of the same strong group. Since all of these couplings involve exotic fields which will be shown to obtain large masses, these dynamical superpotential terms are irrelevant for the infrared theory, and we will not be concerned with them any further.

\subsubsection{Yukawa couplings}

In order to generate the Yukawa couplings we need couple the preons of the two strong groups to the Higgs doublets. This is achieved by introducing the following additional fields, all of which are taken to have masses above the confinement scale.

\begin{tabular}{c|cc|rrrrr} 
field & $S p(6)_{1}$ & $S p(6)_{2}$ & $S U(3)$ & $S U(2)$ & $U(1)_{Y}$ & $U(1)_{B}$ & $U(1)_{L}$ \\
\hline$v_{u}$ & $\square$ & $\square$ & 1 & 1 & 0 & 0 & 0 \\
$v_{d}$ & $\square$ & $\square$ & 1 & 1 & 0 & 0 & 0 \\
$v_{s}$ & $\square$ & $\square$ & 1 & 2 & $-1 / 2$ & 0 & 0 \\
$\bar{v}_{s}$ & $\square$ & $\square$ & 1 & 2 & $1 / 2$ & 0 & 0 \\
$w_{u}$ & $\square$ & 1 & 1 & 1 & $1 / 2$ & $1 / 2$ & $1 / 2$ \\
$\bar{w}_{u}$ & $\square$ & 1 & 1 & 1 & $-1 / 2$ & $-1 / 2$ & $-1 / 2$ \\
$w_{d}$ & $\square$ & 1 & 1 & 1 & $-1 / 2$ & $1 / 2$ & $1 / 2$ \\
$\bar{w}_{d}$ & $\square$ & 1 & 1 & 1 & $1 / 2$ & $-1 / 2$ & $-1 / 2$
\end{tabular}

The gauge symmetries not only allow masses for the new fields but also renormalizable couplings to the preons of table (5.16). We assume a perturbative superpotential of the form

$$
\begin{aligned}
W_{\text {tree }} & =\frac{1}{2}\left(\mu-\alpha_{u}\left(a_{1}+a_{2}\right)\right) v_{u}^{2}+\frac{1}{2}\left(\mu-\alpha_{d}\left(a_{1}+a_{2}\right)\right) v_{d}^{2}+\left(\mu-\alpha_{s}\left(a_{1}+a_{2}\right)\right) \bar{v}_{s} v_{s} \\
& +\mu w_{u} \bar{w}_{u}+\mu w_{d} \bar{w}_{d}+\beta_{1} v_{u}\left(p_{1} p_{2}+r_{1} s_{2}\right)+\beta_{2} v_{d}\left(p_{1} p_{2}+r_{1} s_{2}\right)+\beta_{3} v_{s} q_{1} q_{2} \\
& +\beta_{4} \bar{v}_{s} q_{1} r_{2}+\beta_{5} v_{u} w_{u} r_{2}+\beta_{6} v_{u} w_{d} q_{2}+\beta_{7} v_{d} w_{d} q_{2}+\beta_{8} \bar{w}_{u} H_{u} q_{1}+\beta_{9} \bar{w}_{d} H_{d} q_{1}
\end{aligned}
$$

These couplings communicate between the different sectors and generate masses and Yukawa couplings below the mass scales of the heavy fields and confinement. The superpotential eq. (5.19) is not the most general allowed by the symmetries; to simplify the analysis, we have identified the masses of the $v$ and $w$ fields, and left out a few couplings. Superpotentials are well known to be non-generic, thus leaving out terms which are allowed by symmetries is natural in a supersymmetric theory. We will find that in this slightly simplified version of the model we can get very close to fitting all the masses and angles of the SM with only a few effective parameters, the theory 


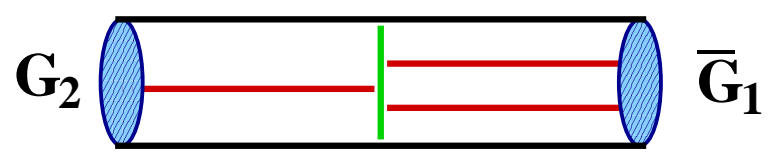

Fig 4. Contribution to the mass of $G, \bar{G}$ in the effective superpotential eq. (5.20). The internal (green) line is the propagator of a heavy $v_{u}$ or $v_{d}$ field and the (black) lines at the top and bottom of the diagram are massless preons which carry SM quantum numbers and are bound into composites together with SM gauge neutral preons (red) by the confining dynamics (blue). The mass generation for the $R, \bar{R}$ fields proceeds through similar diagrams, while the $S, \bar{S}$ mass arises from $v_{s}, \bar{v}_{s}$ exchange.

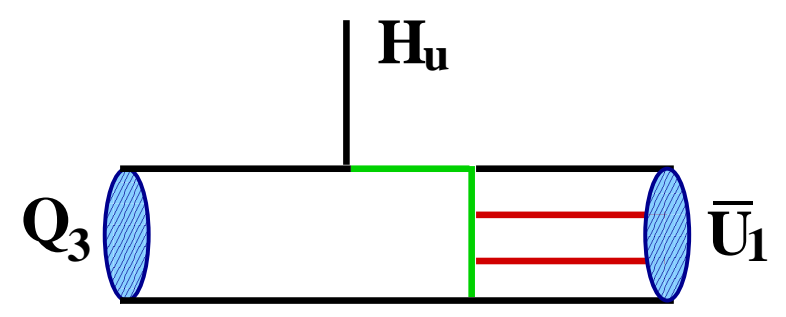

Fig 5. Contribution to SM Yukawa coupling $Y_{31}^{u}$. Internal (green) lines correspond to massive $v$ and $w$ propagators, black (red) lines are preons which carry (do not carry) SM quantum numbers and are bound into composites by the nonperturbative strong dynamics (blue). The Higgs fields in this model are fundamental and have perturbative couplings to preons.

with a generic superpotential whose analysis we do not describe here, has more free parameters entering the Yukawa matrices and can be made fully realistic.

To understand the origin of the SM Yukawa couplings we follow the procedure described in the toy model in $\S 4$ : first integrate out the massive $v$ and $w$ fields at the scale $\mu$ and expand the effective superpotential to dimension 4 in the preon fields $p, q, r, s$, and to dimension 2 in each of the $a$ preons. As in the toy model, below the mass of the $v$ and $w$ 's the gauge couplings of the $S p(6)$ groups evolve rather quickly and get strong at scale $\Lambda$. Preons are confined into composites, and the superpotential is mapped onto an effective superpotential for the composites which contains mass terms for the exotics (Fig 4.) and the desired Yukawa couplings for SM quarks and leptons (Fig 5.)

$$
\begin{aligned}
W_{e f f}= & M_{i j}^{G} G_{i} \bar{G}_{j}+M_{i j}^{R} R_{i} \bar{R}_{j}+M_{i j}^{S} S_{i} \bar{S}_{j} \\
& +Y_{i j}^{u} Q_{i} \bar{U}_{j} H_{u}+Y_{i j}^{d} Q_{i} \bar{D}_{j} H_{d}+Y_{i j}^{l} L_{i} \bar{E}_{j} H_{d}+Y_{i j}^{n} L_{i} \bar{N}_{j} H_{u} .
\end{aligned}
$$

The masses and Yukawa couplings of the effective theory are readily computed in 
terms of the small parameters

$$
\epsilon_{u}=\alpha_{u} \frac{\Lambda}{\mu}, \quad \epsilon_{d}=\alpha_{d} \frac{\Lambda}{\mu}, \quad \epsilon_{s}=\alpha_{s} \frac{\Lambda}{\mu} .
$$

The mass matrices for the exotic fields, $M^{G}, M^{G}$ and $M^{S}$ are

$$
\begin{aligned}
M^{G}=M^{R} & =\frac{\Lambda^{2}}{\mu}\left[\beta_{1}^{2}\left(\begin{array}{ccc}
6 \epsilon_{u}^{4} & 3 \epsilon_{u}^{3} & \epsilon_{u}^{2} \\
3 \epsilon_{u}^{3} & 2 \epsilon_{u}^{2} & \epsilon_{u} \\
\epsilon_{u}^{2} & \epsilon_{u} & 1
\end{array}\right)+\beta_{2}^{2}\left(\begin{array}{ccc}
6 \epsilon_{d}^{4} & 3 \epsilon_{d}^{3} & \epsilon_{d}^{2} \\
3 \epsilon_{d}^{3} & 2 \epsilon_{d}^{2} & \epsilon_{d} \\
\epsilon_{d}^{2} & \epsilon_{d} & 1
\end{array}\right)\right], \\
M^{S} & =\frac{\Lambda^{2}}{\mu} \beta_{3} \beta_{4}\left(\begin{array}{ccc}
6 \epsilon_{s}^{4} & 3 \epsilon_{s}^{3} & \epsilon_{s}^{2} \\
3 \epsilon_{s}^{3} & 2 \epsilon_{s}^{2} & \epsilon_{s} \\
\epsilon_{s}^{2} & \epsilon_{s} & 1
\end{array}\right) .
\end{aligned}
$$

When these fields are integrated out of the theory, they will induce dimension 5 operators with interesting flavor structure into the effective superpotential. For example, integrating out the $G$ and $R$ fields respectively gives rise to operators such as

$$
\frac{1}{M^{G}} Q L \bar{U} \bar{E} \text { and } \frac{1}{M^{G}} Q Q \bar{D} \bar{U}
$$

The flavor structure of these operators is intricate due to the hierarchical nature of the mass matrices (5.22) and the structure of the instanton induced couplings between the exotic and ordinary fields.

The Yukawa matrices for the quarks and leptons (renormalized at the compositeness scale) are given by

$$
\begin{gathered}
Y^{u}=\lambda_{u}\left(\begin{array}{ccc}
6 \epsilon_{u}^{4} & 3 \epsilon_{u}^{3} & \epsilon_{u}^{2} \\
3 \epsilon_{u}^{3} & 2 \epsilon_{u}^{2} & \epsilon_{u} \\
\epsilon_{u}^{2} & \epsilon_{u} & 1
\end{array}\right), Y^{d}=\lambda_{d}\left[\left(\begin{array}{ccc}
6 \epsilon_{d}^{4} & 3 \epsilon_{d}^{3} & \epsilon_{d}^{2} \\
3 \epsilon_{d}^{3} & 2 \epsilon_{d}^{2} & \epsilon_{d} \\
\epsilon_{d}^{2} & \epsilon_{d} & 1
\end{array}\right)+r\left(\begin{array}{ccc}
6 \epsilon_{u}^{4} & 3 \epsilon_{u}^{3} & \epsilon_{u}^{2} \\
3 \epsilon_{u}^{3} & 2 \epsilon_{u}^{2} & \epsilon_{u} \\
\epsilon_{u}^{2} & \epsilon_{u} & 1
\end{array}\right)\right](5.20 \\
Y^{n}=\lambda_{n}\left(\begin{array}{ccc}
6 \epsilon_{u}^{4} & 3 \epsilon_{u}^{3} & \epsilon_{u}^{2} \\
3 \epsilon_{u}^{3} & 2 \epsilon_{u}^{2} & \epsilon_{u} \\
\epsilon_{u}^{2} & \epsilon_{u} & 1
\end{array}\right), Y^{l}=\lambda_{l}\left[\left(\begin{array}{ccc}
6 \epsilon_{d}^{4} & 3 \epsilon_{d}^{3} & \epsilon_{d}^{2} \\
3 \epsilon_{d}^{3} & 2 \epsilon_{d}^{2} & \epsilon_{d} \\
\epsilon_{d}^{2} & \epsilon_{d} & 1
\end{array}\right)+s\left(\begin{array}{ccc}
6 \epsilon_{u}^{3} & \epsilon_{u}^{2} \\
3 \epsilon_{u}^{3} & 2 \epsilon_{u}^{2} & \epsilon_{u} \\
\epsilon_{u}^{2} & \epsilon_{u} & 1
\end{array}\right)\right](5.2
\end{gathered}
$$

The $\lambda$ factors contain an overall $(\Lambda / \mu)^{2}$ times products of the $\beta$ couplings from the ultraviolet superpotential (5.19); $r$ and $s$ are functions of the couplings as well. Note that in order for the top Yukawa coupling to be sufficiently large, $\Lambda \sim \mu$ or large $\beta$ couplings are required.

\subsubsection{Numerical predictions for masses and angles}

From (5.24), one might expect to obtain predictions for four of the seven quark mass ratios and mixing angles because the mass ratios and angles only seem to depend on the three parameters $\epsilon_{u}, \epsilon_{d}$ and $r$. However, to predict the quark and lepton masses

and mixing angles at the weak scale one needs to calculate the renormalization group 
evolution of the couplings from the scale of confinement to the masses of the quarks. The running depends sensitively on the value of the top Yukawa coupling as well as the scale of confinement. At one loop these contributions can be summarized in a single parameter [14] so that we only lose one prediction.

A crude fit to $m_{d} / m_{s}, m_{s} / m_{b}, V_{c b}, m_{c} / m_{t}$ yields the following values for the parameters

$$
\epsilon_{u} \sim 0.045, \quad \epsilon_{d} \sim 0.22, \quad r \sim 4.8
$$

These values can then be used to predict

$$
m_{c} / m_{u}=490, \quad V_{u s}=0.25, \quad \frac{V_{u b}}{V_{c b}}=0.17 .
$$

Whereas these numbers are not in complete agreement with experiment, they are nevertheless encouragingly close. This suggests that the textures obtained in eq. (5.24) might be interesting to study in their own right. In the context of this model, the precise numbers for the predictions above should not be taken too seriously because wave function renormalization for the composites arising from the unknown Kähler potential can alter these predictions by factors presumed to be $\mathcal{O}(1)$.

In the charged lepton sector the model has one more parameter $s$ to describe mass ratios. Fitting to $m_{e} / m_{\mu}$ yields a prediction for $m_{\mu} / m_{\tau}$ that is a factor 1.4 too large. The fit can be improved while avoiding fine-tuning by including an additional set of intermediate fields $w_{l}, \bar{w}_{l}$ which couple to the constituents of the charged leptons.

Neutrino masses can be treated in the same manner as discussed above in §5.1.5. We introduce an extra massive field $w_{N}$ with lepton number one which transforms as an antisymmetric tensor of the $S p(6)_{2}$ group and include the following superpotential couplings

$$
W_{N}=\frac{1}{2}\left(\mu-\alpha_{N} a_{2}\right) w_{N}^{2}+w_{N} r_{2} s_{2}
$$

This interaction gives rise to the Majorana mass matrix (5.9) for the right-handed neutrinos, and after they are integrated out of the theory, the left-handed neutrinos develop hierarchical masses with mass ratios of order $\epsilon_{u}^{4} / \epsilon_{N}^{2}$ through the seesaw mechanism. Since the overal size $\lambda_{n}$ of the Dirac neutrino masses in eq. (5.24) is unconstrained, neutrino mass bounds do not place any constraint on the scale of compositeness.

\subsubsection{Comments}

In summary, this model based on $S p(6)^{2}$ is successful at explaining the existence of three families and in reproducing the observed flavor properties of quarks and

leptons. A great virtue of the model is that it possesses a baryon symmetry, so that 
the compositeness scale need not be high. Constraints on the scale of compositeness will therefore come from flavor changing operators, such as lepton flavor violating operators in the superpotential eq. (5.23) or in the Kähler potential. An investigation of such effects will be pursued elsewhere; here we simply stress that compositeness effects may show up in exotic flavor violating processes.

\section{Conclusions and Outlook}

The new dynamical approach to the flavor problem that we are advocating has been shown to be capable of reproducing the fermion masses and mixing angles seen in Nature, as well as explaining the replication of families. We emphasize that we were able to demonstrate, entirely in the context of renormalizable field theories, how compositeness for quarks and leptons can give rise to realistic flavor structure at low energies. By not resorting to "Planck slop" or other high dimension operators, we have not hidden any of the dynamics responsible for flavor.

In the examples we presented, the three generations of quarks and leptons are composites of new strong interactions. The Yukawa couplings arise from a combination of perturbative dynamics above the scale where the new gauge interactions get strong and confinement. We obtain new textures of Yukawa matrices (4.12) without zeros (but with calculable combinatoric factors) which can be predictive and sufficiently rich to be realistic. The more realistic models we considered in $\$ 5$ are not unifiable, but as demonstrated by our toy model of $\S 4$, the flavor mechanism we are proposing here is at least in principle compatible with unification.

There are a number of avenues to explore from here:

1. Other strong groups. In this paper we have focused on the gauge group $S p(6)$ with an antisymmetric tensor matter field to generate three generations. There are many other models which might be useful for generating composite generations. A promising example is supersymmetric $S U\left(N_{c}\right)$ gauge theory with an adjoint matter field $A$ and $N_{f}$ flavors $Q+\bar{Q}$. Gauge invariants of this theory include the composite operators $M_{j}=Q A^{j} \bar{Q}$ for $j=0,1,2, \cdots$. The dynamics of these theories is not as well understood, but with an added tree level superpotential $W_{\text {tree }}=\operatorname{tr}^{\mathrm{k}+1}$ the theory has a dual description in terms of an $S U\left(k N_{c}-N_{f}\right)$ gauge group [15], and is believed to confine and generate $k$ generations of composites $M_{j}$ for the special case $k N_{c}-N_{f}=1$. Other models can be constructed using $S O, S U, S p$ groups with various tensor matter fields [5, 16].

2. SUSY breaking. The models presented in this paper are incomplete as they do not address the issue of SUSY breaking and electroweak symmetry breaking. It is desirable to have the nonperturbative scale of flavor physics simultaneously explain the electroweak hierarchy. However, relating flavor physics and 
SUSY breaking runs the risk of generating large flavor changing neutral currents through nondegeneracy of squark and slepton masses. To avoid large flavor changing effects, it seems worthwhile to see if the strong flavor dynamics we envision could trigger SUSY breaking in a low energy gauge-mediated model [17]. An alternative would be to try to realize our flavor mechanism within the Effective SUSY scenario [12], in which dangerous flavor changing processes are suppressed by having first and second family sparticles be much heavier than those of the third family. A technical problem is to find a model which breaks SUSY while preserving a large enough non-Abelian global symmetry into which the SM gauge group can be imbedded. Conventional models of dynamical SUSY breaking are constructed by lifting all classical flat directions with a tree level superpotential in a theory in which quantum effects push the vacuum away from the origin, resulting in non-zero vacuum energy and SUSY breaking [18]. These models are unsatisfactory for our purposes since both the tree level superpotential and the VEVs break the desired global symmetries. Recently, models of dynamical SUSY breaking with sufficiently large global symmetries have been constructed [19, 20]; a common feature of these models are classical flat directions which are only lifted by quantum dynamics.

3. Phenomenology. It seems worth pursuing the phenomenology of flavor changing interactions that result from compositeness, such as those discussed briefly in the previous section. It is not clear, however, how much can be said in a model without SUSY breaking. Another feature of phenomenological interest in the models we have described is the ubiquity of neutral moduli such as the $T_{k}=\operatorname{tr} A^{k}$ composite fields. While they can be expected to develop mass when SUSY is broken, there may be many such fields, with flavor dependent couplings, which could in principle mediate detectable long range forces [21].

\section{Acknowledgements}

We are grateful to Ann Nelson for useful conversations. D.B.K and F.L. are supported in part by DOE grant DOE-ER-40561, and NSF Presidential Young Investigator award PHY-9057135. M.S. is supported by the U.S. Department of Energy under grant \#DE-FG02-91ER40676.

\section{References}

[1] H.P. Nilles, Phys. Rep. 110, 1 (1984).

[2] N. Seiberg, Phys. Rev. D49, 6857 (1994), hep-th/9402044; Nucl. Phys. B435, 129 (1995), hep-th/9411149. 
[3] for reviews see e.g. K. Intriligator and N. Seiberg, Nucl. Phys. Proc. Suppl. 45BC, 1 (1996), hep-th/9509066; M.E. Peskin, hep-th/9702094.

[4] M. Strassler, Phys. Lett. 376B, 119 (1996), hep-ph/9510342; A. Nelson and M. Strassler, hep-ph/9607362; M.A. Luty and R.N. Mohapatra, Phys. Lett. 396B, 161 (1997), hep-ph/9611343.

[5] C. Csaki, M. Schmaltz, W. Skiba, Phys. Rev. Lett. 78, 799 (1997), hepth/9610139; hep-th/9612207.

[6] C.D. Carone, L.J. Hall and T. Moroi, hep-ph/9705383.

[7] P. Cho and P. Kraus, Phys. Rev. D54, 7640 (1996), hep-th/9607200.

[8] C. Csáki, M. Schmaltz and W. Skiba, Nucl. Phys. B487, 128 (1997), hepth/9607210.

[9] C.D. Froggatt and H.B. Nielsen, Nucl. Phys. B147, 277 (1979).

[10] Y. Nir and N. Seiberg, Phys. Lett. 309B, 337 (1993), hep-ph/9304307; M. Leurer, Y. Nir and N. Seiberg, Nucl. Phys. B398, 319 (1993), hep-ph/9212278; M. Leurer, Y. Nir and N. Seiberg, Nucl. Phys. B420, 468 (1994), hep-ph/9310320.

[11] P. Pouliot and N. Seiberg, Phys. Lett. 318B, 169 (1993), hep-ph/9308363; D.B. Kaplan and M. Schmaltz, Phys. Rev. D49, 3741 (1994), hep-ph/9311281; P.H. Frampton and O.C.W. Kong Phys. Rev. D53, 2293 (1996), hep-ph/9511343; R. Barbieri, G. Dvali and L.J. Hall, Phys. Lett. 377B, 76 (1996), hep-ph/9512388; K.S. Babu and S.M. Barr; Phys. Lett. 387B, 87 (1996); hep-ph/9606384.

[12] A. Cohen, D. Kaplan and A. Nelson, Phys. Lett. 388B, 588 (1996), hep$\mathrm{ph} / 9607394$.

[13] S.L. Glashow, Published in Providence Grand Unification 88 (1984).

[14] S. Dimopoulos, L.J. Hall and S. Raby, Phys. Rev. Lett. 68, 1984 (1992); V. Barger, M.S. Berger and P. Ohmann, Phys. Rev. D47, 1093 (1993), hepph/9209232; Phys. Rev. D47, 2038 (1993), hep-ph/9210260.

[15] D. Kutasov, Phys. Lett. 351B, 230 (1995), hep-th/9503086; D. Kutasov and A. Schwimmer, Phys. Lett. 354B, 315 (1995), hep-th/9505004; D. Kutasov, A. Schwimmer, and N. Seiberg, Nucl. Phys. B459, 455 (1996), hep-th/9510222.

[16] K. Intriligator, Nucl. Phys. B448, 187 (1995), hep-th/9505051; K. Intriligator, R. Leigh, and M. Strassler, Nucl. Phys. B456, 567 (1995), hep-th/9506148; J. Brodie and M. Strassler, hep-th/9611197. 
[17] M. Dine and A. Nelson, Phys. Rev. D48, 1277 (1993), hep-ph/9303230; M. Dine, A. Nelson and Y. Shirman, Phys. Rev. D51, 1362 (1995), hep-ph/9408384; M. Dine, A. Nelson, Y. Nir and Y. Shirman, Phys. Rev. D53, 2658 (1996), hepph/9507378; L. Randall, hep-ph/9612426.

[18] W. Skiba, hep-th/9703159; and references therein.

[19] K. Intriligator and S. Thomas, Nucl. Phys. B473, 121 (1996), hep-th/9603158;

E. Poppitz and S.P. Trivedi, Phys. Rev. D55, 5508 (1997), hep-ph/9609529;

N. Arkani-Hamed, J. March-Russell and H. Murayama, hep-ph/9701286; K.I.

Izawa, Y. Nomura, K. Tobe and T. Yanagida, hep-ph/9705228; H. Murayama, hep-ph/9705271.

[20] A. Nelson, private communication.

[21] S. Dimopoulos and G.F. Giudice, Phys. Lett. 379B, 105 (1996), hep-ph/9602350. 